\title{
Kinetic Studies of the Interaction of Toluidine Blue with Poly- ( $\alpha$ L-glutamic acid) by Means of Stopped-Flow Method
}

\author{
Hidetoshi Ushio, Hiroshi Uchimura, Yoshikuni TsujI, \\ Takayuki SANo, and Tatsuya YasunAGA \\ Department of Chemistry, Faculty of Science, \\ Hiroshima University, Hiroshima 730, Japan.
}

(Received June 9, 1977)

\begin{abstract}
Kinetic and equilibrium studies on the interaction between toluidine blue and poly $(\alpha \mathrm{L}$-glutamic acid $)$ were carried out for both the helical and coiled conformations of polymer. The spectrophotometric measurements show that one bound dye molecule occupies about two and one carboxylic group for the helical and coiled forms, respectively. In the stopped-flow experiments, a relatively fast reaction process was observed for the coiled form, while, a slow process was observed with the helical form. The fast process was attributed to the conformational change of the polymer induced by the dye binding.

KEY WORDS Kinetics / Toluidine Blue / Stopped-Flow / Poly $(\alpha \mathrm{L}$ glutamic acid)/Conformational Change / Extended Form / Compact Form /
\end{abstract}

As a convenient model for the interaction of small ligand with biopolymer, the basic dyemacromolecule system was investigated. ${ }^{1,2}$ It is generally known that the metachromatic effect is induced as a result of the dye-dye interaction on the polymer, which introduces a cooperative nature into the binding of the dye to the polymer. ${ }^{2,3}$ Actually, the complete thermodynamic theory of the cooperative binding has been reported ${ }^{4}$ and has been successfully applied to the several systems of the basic dyes and synthetic polymers. ${ }^{5-7}$

Many kinetic studies on these systems have been performed mainly by means of the temperaturejump method. Most of these, however, have concentrated on the investigation of the fast association-dissociation process in the dye-macromolecule system. ${ }^{6,8,9}$ Recently, the slow process was found in the acridine orange-poly $(\alpha \mathrm{L}$-glutamic acid) ${ }^{10,11}$ and proflavine-poly (acrylic acid) systems. ${ }^{12}$ These slow processes were attributed to the formation process of the dye oligomer on the polymer surface $^{10}$ and the intermacromolecular interaction induced by the dye binding. ${ }^{11}$

In two previous other works, the kinetic properties of the fast binding process of the dye to the polymer were clarified by the toluidine blue-poly$\left(\alpha\right.$, L-glutamic acid) ${ }^{13}$ and poly(acrylic acid) ${ }^{14}$ systems, using the electric-field-pulse method. In the former system, the slow process was also observed by the stopped-flow method but could not be explained by the above two mechanisms.

The purpose of this work is to determine the mechanism for the slow process in this system by the stopped-flow method.

\section{EXPERIMENTAL}

\section{Materials}

The sodium poly $\left(\alpha\right.$ L-glutamic acid), $(\mathrm{Glu})_{n}$, having an average degree of polymerization of 700 was a generous gift provided by Ajinomoto Company Inc. After dialyzing against deionized water for three days, it was lyophilized and dried in vacuum at $45^{\circ} \mathrm{C}$ for $7 \mathrm{hr}$ prior to use.

Toluidine blue was purchased from Chroma Gesellschaft as the chloride salt and was purified according to the procedure of Pal and Schubert. ${ }^{15}$ Subsequently further purification was performed by washing with chloroform until the chloroform layer was colorless. A thin-layer-chromatography analysis showed a single spot. The concentration of the stock solution of dye was determined spectrophotometrically using an extinction coefficient of $\varepsilon_{635}=3.65 \times 10^{4} \mathrm{M}^{-1} \mathrm{~cm}^{-1}$ for a suf- 
ficiently diluted dye solution (around $2 \times 10^{-5} M$ ). Dye solutions were freshly prepared before the experiments. All other chemicals used were of reagent grade.

Experiments were carried out in $1-\mathrm{m} M$ acetate buffer for the helical (Glu) ${ }_{n}$ and in 1-mM phosphate buffer for the coiled form. The $\mathrm{pH}$ values of these buffered sample solutions were fixed at $\mathrm{pH} 4.7$ and 7.5 for the helical and coiled forms, respectively, with an accuracy of $\pm 0.1 \mathrm{pH}$ unit over the polymer to dye molar ratio $(P / D)$ range from 1 to about 20.

In spectrophotometric measurements, the sample solutions were prepared by the dropwise addition of a buffered dye solution to buffered (Glu) solution with stirring. In order to retain the conditions analogous to those in a previous work, ${ }^{13}$ the value of $P / D$ was varied from 1 to 20 and the total dye concentration $\left(c_{\mathrm{A}}{ }^{0}\right)$ was fixed at $2 \times 10^{-5} \mathrm{M}$ in most cases. Compared with the previous work, ${ }^{13}$ use of a buffer in these experiments is just one point of difference in the experimental conditions.

\section{Method}

Spectrophotometric measurements were carried out using the Union Giken SM 401 spectrophotometer. The $\mathrm{pH}$ value was measured with the Hitachi-Horiba type F-5 pH meter.

Kinetic measurements were made using the stopped-flow apparatus whose mixer was purchased from Union Giken, and the other assemblies were made in our laboratory. The dead-time of this apparatus was less than $1 \mathrm{msec}$. In the stoppedflow experiments, a buffered (Glu) $)_{n}$ solution was mixed with an equal volume of buffered toluidine blue solution. The course of the reaction was monitored at $635 \mathrm{~nm}$ which was due to the absorption of toluidine blue monomer. ${ }^{16}$ The range of the total concentration of $(\mathrm{Glu})_{n}\left(c_{\mathrm{P}}{ }^{0}\right)$ was $2-40 \times$ $10^{-5} M$. In the experiments when the $P / D$ is varied, the dye concentration was fixed at $2 \times$ $10^{-5} M$ (after mixing) and the $(\mathrm{Glu})_{n}$ concentration was increased, that is, the $P / D$ was varied up to 20 . Some experiments were also carried out in which the concentrations of both the $(\mathrm{Glu})_{n}$ and the toluidine blue were varied at a constant $P / D$. For the kinetic experiments, the temperature was controlled at $20 \pm 1^{\circ} \mathrm{C}$ by circulating water from an external thermostat. Other experiments were carried out at room temperature $\left(20^{\circ} \mathrm{C}\right)$.

\section{RESULTS AND DISCUSSION}

\section{Spectrophotometric Measurements}

The molar extinction coefficient of the stacked dye $\left(\varepsilon_{\mathrm{st}}\right)$, the number of binding site per segment $(g)$, the cooperative binding constant $(K)$ and the cooperative parameter $(q)$ were determined using Schwarz's theory in which the cooperative binding occurs through the following three processes. Equation 1 is the growth process and the others are nucleation processes, ${ }^{4}$

$$
\begin{aligned}
& \text { auu }+\mathrm{A} \rightleftharpoons \mathrm{aau} \\
& \mathrm{uuu}+\mathrm{A} \rightleftharpoons \mathrm{aau} \\
& \text { aua }+\mathrm{A} \rightleftharpoons \text { aaa }
\end{aligned}
$$

where $u$ and a are an unoccupied binding site and an occupied binding site, respectively and $\mathbf{A}$ is a free monomeric dye. The binding constants for the reactions of (1) and (2) were $K$ and $K^{\neq}$, respectively and the cooperative parameter is defined as $q=K / K^{\ddagger}$.

The fraction of the occupied binding site $(\theta)$ is defined as

$$
\theta=\frac{c_{\Delta}{ }^{0}\left(1-\gamma_{\Delta}{ }^{*}\right)}{g c_{\mathrm{P}}{ }^{0}}
$$

where $\gamma_{A}^{*}$ is a fraction of the free dye.

In this paper, only the results of these parameters are given in Table I.

The values of 0.54 and 1.15 for $g$ are in close agreement with those obtained in our previous work ${ }^{13}$ and this implies that one dye molecule binds to two and one residue of $(\mathrm{Glu})_{n}$ in the helical and the coiled forms, respectively. On the other hand, there is a difference in the values of $q$ for the coiled form in the present and the previous works. This can be explained as follows. In a previous work, the value of $q$ was determined in the region near $\theta=1 / 2$, at which the theory does not hold, so that the value of $q$ would become small. This is also pointed out by Schwarz and Klose. ${ }^{12}$ However, in the present case, the value of $q$ was determined in a sufficiently large $P / D$ region and thus turned out to be a large value of 130 which is reasonable compared with the $q$ values in other polymer-dye systems. $^{5,6,12}$

It is generally accepted that cooperativity results from the interaction between dyes on the polymer and that it increases with an increase in the overlap- 
Table I. Equilibrium and kinetic parameters of toluidine blue- $\operatorname{poly}(\alpha$, L-glutamic acid $)$ system $^{2}$

\begin{tabular}{ccccccc}
\hline $\mathrm{pH}$ & $\begin{array}{c}\varepsilon_{\mathrm{st}} \\
10^{3} M^{-1} \mathrm{~cm}^{-1}\end{array}$ & $g$ & $\begin{array}{c}K, \\
10^{5} M^{-1}\end{array}$ & $q$ & $\begin{array}{c}k_{1}, \\
10^{2} \mathrm{sec}^{-1}\end{array}$ & $\begin{array}{c}k_{-1}, \\
\mathrm{sec}^{-1}\end{array}$ \\
\hline 4.7 & $5.3( \pm 0.3)$ & $0.54( \pm 0.08)$ & 1.5 & 2 & & \\
{$[5.0]$} & & {$[0.45( \pm 0.04)]$} & {$[1.4( \pm 0.3)]$} & {$[3]$} & & \\
7.5 & $5.6( \pm 0.1)$ & $1.15( \pm 0.05)$ & $7.1( \pm 0.6)$ & 130 & $4.6( \pm 0.3)$ & $1( \pm 2)$ \\
{$[8.0]$} & & {$[1.1( \pm 0.04)]$} & {$[6.9( \pm 0.5)]$} & {$[8]$} & & \\
\hline
\end{tabular}

a The square brackets indicate the data from our previous work. ${ }^{13}$

ping area between the stacked dyes. ${ }^{2,4}$ From the fact that the value of $g$ in the coiled form is greater than that in the helical form, it is suggested that the extent of interaction between dyes is more predominant in the coiled form than in the helical form. This seems to be the reason for the difference in the value of $q$ in the two forms.

\section{Stopped-Flow Experiments}

Typical kinetic traces are shown in Figure 1. In all the kinetic experiments, the reaction proceeded with an increase in the transmittance at $635 \mathrm{~nm}$. Furthermore, from another experiment,

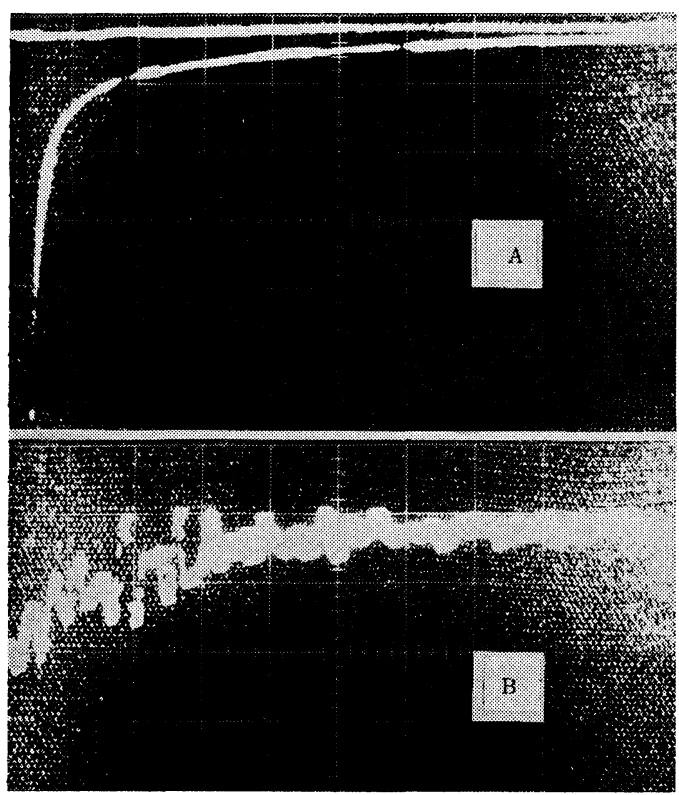

Figure 1. Typical kinetic traces for the interaction of toluidine blue $(40 \mu M)$ with $\operatorname{poly}(\alpha, \mathrm{L}$-glutamic acid) $(200 \mu M)$ at the wavelength of $635 \mathrm{~nm}$ : (A) in acetate buffer, $\mathrm{pH} 4.8$, the vertical scale is 0.1 volt/division, and the horizontal scale is $5 \mathrm{sec} /$ division; (B) in phosphate buffer, $\mathrm{pH} 7.5$, the vertical scale is $20 \mathrm{mV} /$ division, and the horizontal scale is $10 \mathrm{msec} /$ division. it was found that there is a considerably large change in the transmittance within the dead-time of the instrument. As shown in Figure 1, a relatively fast process was observed in the coiled form. In the helical form, however, both the fast and slow processes were observed. In order to examine the origin of the difference in these reaction processes, the following experiment was performed. The buffered sample solutions having the values of $\mathrm{pH}$ from 4.7 to 5.9 and 5.8 to 7.6 were prepared using 1-m $M$ acetate and phosphate buffers, respectively, and the stopped-flow experiments were carried out with these solutions. As a result, there was no difference in the two signals at the same $\mathrm{pH}$ for the acetate buffered and the phosphate buffered solutions. It was also found that the fast process appeared above the $\mathrm{pH}$ of the helix-coil transition and that the slow process appeared below that $\mathrm{pH}$. According to these findings, it can thus be shown that the difference in the reaction process between $\mathrm{pH} 4.7$ and 7.5 is caused by the transition in the conformation of the polymer.

The kinetic data of the fast reaction process in the coiled form fit a single exponential equation, suggesting that this process is a first-order reaction. In the case of helical form, it is not certain as to whether the observed signal contains two kinds of reaction or one kind of the second-order reaction, and also as to, whether the fast process of this signal is the same as that in the coiled form or not, since this process could not be analyzed independently. However, from the fact that the time region, in which the fast process in the helical form was observed, differed from that in the coiled form by one order of magnitude, it may be supposed that the fast process in the helical form differs in kind from that in the coiled form. Therefore, in the helical form, only the slow process was analyzed and the relaxation time could be defined only close 


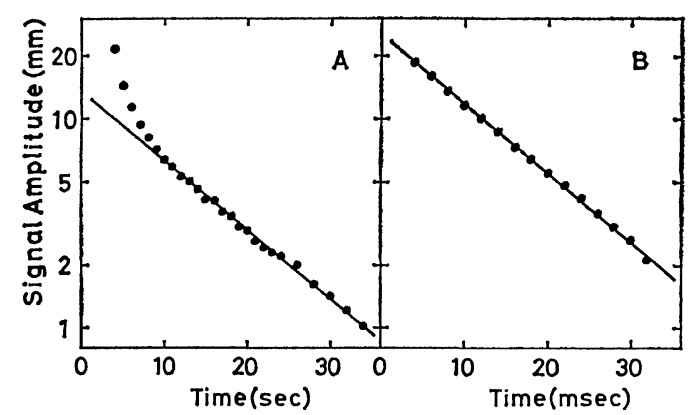

Figure 2. Semi-logarithmic plots of the signal in Figure 1 vs. time using arbitrary units.

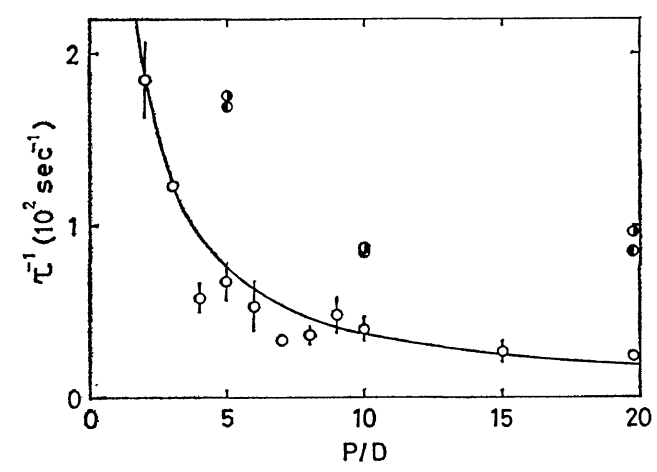

Figure 3. Plots of the reciprocal relaxation time, $(1 / \tau)$, vs. polymer to dye ratio, $P / D$, at constant total concentration of the dye $(20 \mu M)$ in phosphate buffer, pH 7.5: (O) $\mathrm{NaCl}$ concentration of $0 \mathrm{mM}$; () $5 \mathrm{mM}$; (ब) $10 \mathrm{~m} M$. The solid line was calculated using eq 6 .

to equilibrium. The semilogarithmic plots of these signals in Figure 1 are shown in Figure 2. From the differences in the value of relaxation time and its dependence on $c_{\mathrm{A}}{ }^{\circ}$ between the two forms, it is expected that the mechanism of the fast reaction is probably different from that of the slow one. Therefore, in this paper, only the fast process in the coiled form was analyzed.

Figures 3 and 4 show the relaxation time plots obtained against $P / D$ and $c_{\mathrm{A}}^{\circ}$, respectively. From these figures, it can be seen that the relaxation time increases significantly with an increase in $P / D$ at constant dye concentration, but that it is independent of both the concentration of $(\mathrm{Glu})_{n}$ and that of toluidine blue at constant $P / D$ within experimental error. Therefore, based on the behavior of the relaxation time, certain reactions are proposed as a possible mechanism.

Cooperative Binding Process. In a previous

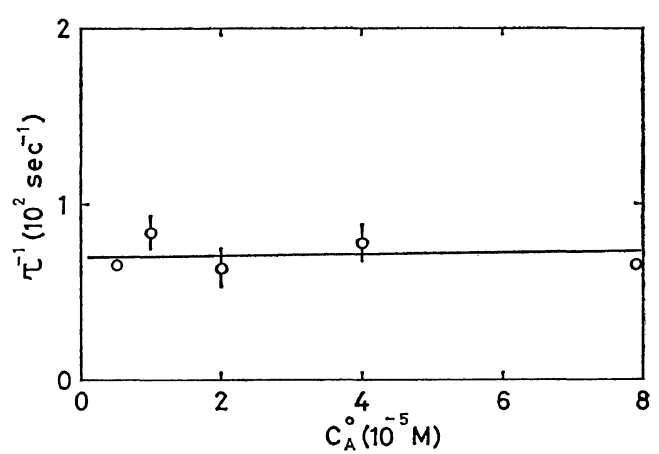

Figure 4. Plot of the reciprocal relaxation time, $(1 / \tau), v s$. total concentration of the dye, $c_{\mathrm{A}}{ }^{\circ}$, at constant polymer to dye ratio $(P / D=5)$ in phosphate buffer, $\mathrm{pH}$ 7.5. The solid line was calculated using eq 6 .

work, the relaxation time for the reaction of eq 1 was found to be about $10^{-4} \mathrm{sec}-10^{-5} \mathrm{sec}$, which is two or one order of magnitude smaller than that for the present reaction. Therefore, the reaction mechanism of eq 1 can not apply to the present reaction. With respect to eq 2 and 3 , there is very very little possibility of observing these reactions since the concentrations of uau and aua are small compared with those of auu and aau. These reactions, however, are coupled to each other and their relaxation equation is very complicated, making the quantitative assignment difficult. Therefore, from another standpoint, it is considered that these reaction mechanisms may or may not be suited to the present reaction. The concentrations of uau and aua become large in the helical form because of the small $q$ value, so that the reactions of eq 2 and 3 become more predominant in comparison with those in the coiled form. However, this is inconsistent with the fact that the present reaction could not be observed in the helical form. From these facts, the mechanism of eq 2 and 3 does not apply to the present experimental results.

Intermacromolecular Interaction. Schwarz and Klose found that the slow relaxation process in addition to the fast process was due to the dye redistribution process in proflavine-poly(acrylic acid) system. ${ }^{12}$ They suggested that the intermacromolecular interaction induced by the bound dye is the most likely mechanism for this slow process. Therefore, an examination was made to determine if this interaction was applicable to the present experimental results. 
The intermacromolecular interaction induced by the bound dye seems to be affected by the following two factors. One is the polymer concentration concerned with the number of collision. The other is the degree of neutralization of the residue whose increase makes the electric repulsive force between the polymers weak and subsequently collision occurs easily. With increasing polymer concentration at constant dye concentration, the degree of neutralization decreases since the bound dyes are distributed among the polymers. Thus, the experiment with varying $P / D$ at constant dye concentration involves contrary contributions to the intermacromolecular interaction and so this mechanism is not applicable to it. On the other hand, by increasing both the polymer and dye concentrations at constant $P / D$, the degree of neutralization of residue will be held nearly canstant and then the reciprocal relaxation time may be expected to increase. As shown in Figure 4, however, no dependence of the reciprocal relaxation time on the total dye concentration was observed within experimental error. Consequently, the intermacromolecular interaction induced by the bound dye seems improbable.

Conformational Change of the Polymer Induced by the Dye Binding

The polyelectrolyte, such as poly(methacrylic acid), could take on an extended conformation brought on by the electric repulsive force between the ionized residues. ${ }^{17,18}$ Then, by decreasing the degree of ionization $(\alpha)$, the polymer changes its conformation from an extended to a compact one. Since the bound dye neutralizes the negative charge of the polymer, this kind of conformational change may also be expected to be induced by the dye binding, ${ }^{17,18}$ and to proceed as a first-order reaction, and this is consistent with the experimental results. On the other hand, in the helical form of (Glu) ${ }_{n}$, this kind of reaction can not occur because of the rigid sturcture of the $\alpha$-helix. This fact also serves to interpretes successfully the experimental results that this reaction process as observed in the coiled form was not observed in the helical form. Thus, this mechanism seems the most plausible for the present relaxation phenomena.

In simplist form, this reaction is expressed as follows

$$
\text { Extended form } \underset{k_{-1}}{\stackrel{k_{1}}{\rightleftharpoons}} \text { Compact form }
$$

The rate of this reaction is affected by the electric charges on the polymer which can be expressed as $(\alpha-g \theta)$. Therefore, assuming that the forward and backward reactions occur where the residues are neutralized and ionized, respectively, the kinetic equation for eq 4 may be written

$$
-\frac{\mathrm{d} c_{\mathrm{e}}}{d t}=k_{1}\{1-(\alpha-g \theta)\} c_{\mathrm{e}}-k_{-1}(\alpha-g \theta) c_{\mathrm{c}}
$$

where $c_{\mathrm{e}}$ and $c_{\mathrm{c}}$ are the polymer concentration of the extended and compact forms, respectively. If the value of $\theta$ is constant throughout the reaction, the relaxation time may be expressed as eq 6 .

$$
\begin{aligned}
\tau^{-1} & =k_{1}\{1-(\alpha-g \theta)\}+k_{-1}(\alpha-g \theta) \\
& =\left(k_{-1}-k_{1}\right)(\alpha-g \theta)+k_{1}
\end{aligned}
$$

Figure 5 shows the polts of eq 6 in the experiment in which $P / D$ was varied at constant $c_{\Delta}^{\circ}$. The values of $(\alpha-g \theta)$ were calculated using the values of $g$ and $\theta$ obtained spectrophotometrically and the value of $\alpha$ found in the literature. As shown in Figure 5, a good linearity was obtained and the values of $k_{1}$ and $k_{-1}$ were determined as $455 \mathrm{sec}^{-1}$ and $1 \mathrm{sec}^{-1}$, respectively. Next, the reciprocal relaxation times were calculated by eq 6 , using these $k_{1}$ and $k_{-1}$ values at the various dye concentrations under a constant $P / D$ these are shown by the solid line in Figure 4. A sufficiently good agreement may be seen between the experimental and calculated values, implying that the present relaxation phenomena can be assigned to the conformational change of the polymer.

Furthermore, in order to ascertain the model as

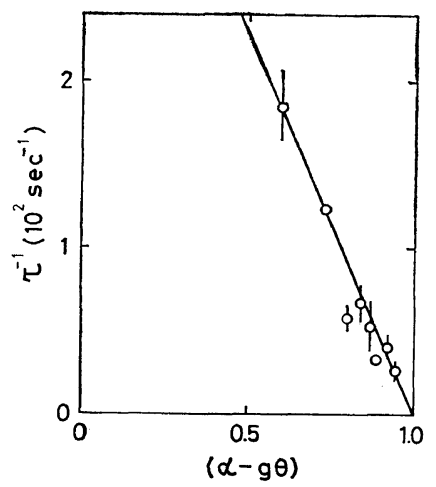

Figure 5. Plots of the reciprocal relaxation time, $(1 / \tau)$, vs. $(\alpha-g \theta)$ under the same experimental condition as that in Figure 3. The solid line was drawn as the bestfit line through the data. 


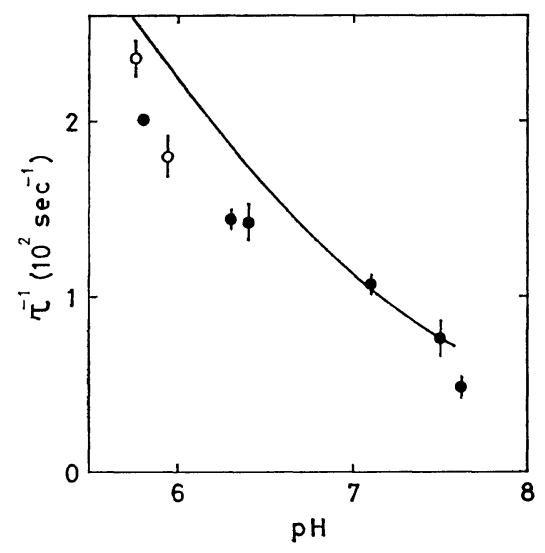

Figure 6. Plots of the reciprocal relaxation time, $(1 / \tau), v s . \mathrm{pH}$ at constant polymer to dye ratio $(P / D=5)$ and total concentration of the dye $(20 \mu M)$ : (O) in acetate buffer; (๑) in phosphate buffer. The solid line was calculated according to eq 6 .

proposed above, several experiments were carried out. First, the dependence of the relaxation time on the $\mathrm{pH}$ was examined. The results are shown in Figure 6. A fair agreement may be seen in the tendency of the reciprocal relaxation times to decrease with increasing $\mathrm{pH}$, but there is considerable difference between the experimental and calculated values, especially below $\mathrm{pH}$ 7. This may be explained as follows. In this $\mathrm{pH}$ region, $(\mathrm{Glu})_{n}$ forms a helical conformation which tends to prevent a conformational change. Therefore the value of $k_{1}$ is expected to take on a somewhat smaller value. The value of $\alpha$ used in the calculation was obtained from the data reported by Hatano, et al. ${ }^{20}$ but his experimental conditions are different from ours in that we used a buffer. It is known that by increasing the salt concentration, the midpoint of the titration curve shifts toward the low $\mathrm{pH}$ region and the value of $\alpha$ at certain $\mathrm{pH}$ increases. Therefore, owing to the small value of $\alpha$ used, the calculated value of $\tau^{-1}$ might be resulted in a large value.

Secondly, the dependence of the relaxation time on the salt concentration was examined. It has been reported that a compact conformation becomes more stable by increasing the salt concentration. $^{18,19}$ This suggests that the value of the rate constant will increase in $k_{1}$ and decrease in $k_{-1}$ and consequently the value of $\tau^{-1}$ will become large. The experimental results shown in Figure
3 are consistent with the above expectation.

Finally, the dependence of the relaxation time on the degree of polymerization was examined. The reaction of the conformational change is thought to be related to the chain length of the polymer. For a polymer of short length, the reaction will not occur. Indeed, as a result of the stopped-flow measurement, the no reaction process was observed with $(\mathrm{Glu})_{n}$ to a polymerization degree of 20 .

Thus, the experimental results successfully support the validity of the proposed mechanism of the conformational change of the polymer, but some problems still exist. In the kinetic measurement, the reaction was monitored through a change in the concentration of free monomeric dye apparently not involved in the present mechanism of eq 4. Thus, another mechanism of monitoring the reaction progress may be considered as follows. It is reported that the isolated bound dye has the same extinction coefficient as that of free monomeric dye. ${ }^{5}$ When the extended polymer changes its conformation to the compact one, the isolated bound dye can be brought so close that they interact with each other. This results in a metachromatic effect which increases in the transmittance of free monomeric dye and decreases in that of the metachromatic band. Accordingly, the amount of the bound dye can be held constant during this reaction, this is consistent with the assumption that the value of $\theta$ is constant during this reaction.

Finally, on the basis of these results, the following dynamic feature of the interaction of the dye with the macromolecule may be considered. When the dye is mixed with the polymer, at the first stage, the dye binds to the polymer through the diffusion-controlled reaction of (1) and (2). Then, the negative charge on the polymer is neutralized by the bound dye. At the second stage, the polymer changes its conformation from an extended to a compact form.

\section{REFERENCES}

1. A. R. Peacocke and J. N. H. Skerrett, Trans. Faraday Soc., 52, 261 (1956).

2. D. F. Bradley and M. K. Wolf, Proc. Natl. Acad. Sci. U.S.A., 45, 944 (1959).

3. R. W. Armstrong, T. Kurucsev, and U. P. Strauss, J. Am. Chem. Soc., 92, 3174 (1970).

4. G. Schwarz, Eur. J. Biochem., 12, 442 (1970). 
5. G. Schwarz, S. Klose, and W. Balthasar, ibid., 12, 454 (1970).

6. G. Schwarz and W. Balthasar, ibid., 12, 461 (1970).

7. V. Vitagliano, L. Constantino, and A. Zagari, $J$. Phys. Chem., 77, 204 (1973).

8. G. G. Hammes and C. D. Hubbard, ibid., 70, 1615 (1966).

9. G. G. Hammes and C. D. Hubbard, ibid., 70, 2889 (1966).

10. M. Tsuda, Bull. Chem. Soc. Jpn., 48, 1709 (1975).

11. G. Schwarz and A. Seelig-Löffler, Biochim. Biophys. Acta, 397, 125 (1975).

12. G. Schwarz and S. Klose, Eur. J. Biochem., 29, 249 (1972).

13. H. Ushio, T. Yasunaga, T. Sano, and Y. Tsuji,
Biopolymers, 15, 187 (1976).

14. H. Ushio, H. Nishimura, Y. Tsuji, T. Sano, and T. Yasunaga, Polym. J., 9, 489 (1977).

15. M. K. Pal and M. Schubert, J. Am. Chem. Soc., 84, 4384 (1962).

16. L. Popa and R. Repanovici, Biochim. Biophys. Acta, 182, 158 (1969).

17. W. H. J. Stork, P. L. de Hasseth, G. J. M. Lippits, and M. Mandel, J. Phys. Chem., 77, 1778 (1973).

18. J. S. Tan and R. L. Schneider, ibid., 79, 1380 (1975).

19. J. S. Tan and S. P. Gasper, J. Polym. Sci., 12, 1785 (1974).

20. M. Hatano, M. Yoneyama, and Y. Sato, Biopolymers, 12, 895 (1973). 